\title{
The antianginal ranolazine mitigates obesity-induced nonalcoholic fatty liver disease and increases hepatic pyruvate dehydrogenase activity
}

\author{
Rami Al Batran, ${ }^{1,2,3}$ Keshav Gopal, , ${ }^{1,2,3}$ Hanin Aburasayn, ${ }^{1,2,3}$ Amina Eshreif,, ${ }^{1,2,3}$ Malak Almutairi, ${ }^{1,2,3}$ \\ Amanda A. Greenwell, ${ }^{1,2,3}$ Scott A. Campbell, ${ }^{2,4}$ Bruno Saleme,, ${ }^{3,5}$ Emily A. Court, ${ }^{1}$ Farah Eaton, ${ }^{1,2,3}$ \\ Peter E. Light, ${ }^{2,3,4}$ Gopinath Sutendra, ${ }^{3,5}$ and John R. Ussher ${ }^{1,2,3}$ \\ ${ }^{1}$ Faculty of Pharmacy and Pharmaceutical Sciences, ${ }^{2}$ Alberta Diabetes Institute, ${ }^{3}$ Cardiovascular Research Centre, \\ ${ }^{4}$ Department of Pharmacology, and ${ }^{5}$ Department of Medicine, University of Alberta, Edmonton, Alberta, Canada
}

\begin{abstract}
Obese individuals are often at risk for nonalcoholic fatty liver disease (NAFLD), insulin resistance, type 2 diabetes (T2D), and cardiovascular diseases such as angina, thereby requiring combination therapies for their comorbidities. Ranolazine is a second-line antianginal agent that also improves glycemia, and our aim was to determine whether ranolazine modifies the progression of obesityinduced NAFLD. Twelve-week-old C57BL/6] male mice were fed a low-fat or high-fat diet for 10 weeks and then treated for 30 days with either vehicle control or ranolazine $(50 \mathrm{mg} / \mathrm{kg}$ via daily s.c. injection). Glycemia was monitored via glucose/pyruvate/insulin tolerance testing, whereas in vivo metabolism was assessed via indirect calorimetry. Hepatic triacylglycerol content was quantified via the Bligh and Dyer method. Consistent with previous reports, ranolazine treatment reversed obesity-induced glucose intolerance, which was associated with reduced body weight and hepatic steatosis, as well as increased hepatic pyruvate dehydrogenase (PDH) activity. Ranolazine's actions on hepatic PDH activity may be directly mediated, as ranolazine treatment reduced PDH phosphorylation (indicative of increased PDH activity) in HepG2 cells. Therefore, in addition to mitigating angina, ranolazine also reverses NAFLD, which may contribute to its documented glucose-lowering actions, situating ranolazine as an ideal antianginal therapy for obese patients comorbid for NAFLD and T2D.
\end{abstract}

Authorship note: RAB and KG are co-first authors.

Conflict of interest: The authors have declared that no conflict of interest exists.

License: Copyright 2019, American Society for Clinical Investigation.

Submitted: August 31, 2018 Accepted: November 27, 2018 Published: January 10, 2019

Reference information: JCI Insight. 2019;4(1):e124643. https://doi.org/10.1172/jici. insight.124643.

\section{Introduction}

Nonalcoholic fatty liver disease (NAFLD)/hepatic steatosis is a condition in which excess fat accumulates in the liver of an individual without a history of alcohol abuse, and it is a major risk factor for insulin resistance and type 2 diabetes (T2D) (1,2). Indeed, insulin resistance is the most common disorder associated with NAFLD, regardless of whether the individual is lean or obese. Moreover, both NAFLD and T2D promote dyslipidemia, which increases the risk for cardiovascular diseases, including hypertension, atherosclerosis, and angina pectoris (3). Taken together, patients comorbid for NAFLD, T2D, and cardiovascular disease are likely to receive combination therapy, and it is imperative that we increase our understanding of how combination therapies affect the various pathologies a given patient has. For example, manufacturers of new therapies for $\mathrm{T} 2 \mathrm{D}$ are under intense scrutiny to ensure their medications do not increase cardiovascular risk when compared with standard-of-care $(4,5)$. Conversely, it is often not considered that these T2D patients are also receiving medications for their cardiovascular disease, and our knowledge of the consequences that these cardiovascular medications have on glucose homeostasis and other T2D risk factors — including NAFLD — is limited.

Of interest, the piperazine derivative ranolazine, which is a second-line agent for treating angina via inhibiting the late inward sodium current $\left(\mathrm{I}_{\mathrm{Na}}\right)$ during cardiac repolarization $(6,7)$, may be a more ideal therapy to prescribe in a T2D patient comorbid for angina due to its glycemia-lowering properties. Results from the MERLIN TIMI-36 trial demonstrated that a 4-month treatment with ranolazine (1000 mg twice daily) decreased glycated hemoglobin (HbA1c) levels from $7.5 \%-6.9 \%$ in diabetic subjects (8). Moreover, a prospective trial in T2D patients with $\mathrm{HbA} 1 \mathrm{c}$ levels ranging from $7 \%-10 \%$ treated 
twice daily with $1000 \mathrm{mg}$ ranolazine for 24 weeks observed a greater decline in HbA1c levels versus those treated with placebo control (9). Of interest, ranolazine treatment decreased both fasting insulin and glucagon levels, while improving postprandial glucose and glucagon levels over 3 hours following a mixed-meal tolerance test in these T2D patients (9). Studies in rats suggest that ranolazine may act directly on islet $\alpha$-cells to reduce glucagon secretion (10).

Our current work, to our knowledge, adds a new dimension by which ranolazine improves glycemic control. We observed that a 30-day treatment period with ranolazine was able to improve glycemia in obese mice without impacting skeletal muscle insulin sensitivity. Moreover, ranolazine treatment did not reduce circulating glucagon levels in our study but, instead, resulted in a significant reduction in both overall adiposity and hepatic steatosis. These beneficial actions of ranolazine were associated with increased hepatic pyruvate dehydrogenase $(\mathrm{PDH})$ activity, the rate-limiting enzyme of glucose oxidation (11), consistent with past studies demonstrating that $\mathrm{PDH}$ is a novel target for ameliorating NAFLD $(12,13)$.

\section{Results}

Ranolazine treatment reverses obesity-induced dysglycemia. Treatment with ranolazine for 30 days consistently lowered random-fed blood glucose levels in obese mice, while also improving glucose tolerance (Figure 1, $\mathrm{A}-\mathrm{C})$. The ranolazine-mediated improvement in glucose tolerance could not be attributed to changes in circulating insulin levels at the 0 - and 15-minute time points (Figure 1D). Conversely, ranolazine treatment was devoid of benefit on insulin sensitivity, as both the drop in blood glucose levels and insulin-stimulated Akt/GSK3 $\beta$ phosphorylation in gastrocnemius muscles remained similar between saline- and ranolazine-treated lean or obese mice in response to an insulin tolerance test (Figure 2).

Ranolazine treatment increases energy expenditure. Ranolazine treatment also caused significant reductions in body weight (Figure 3A), primarily attributable to decreases in total adiposity (Figure 3, B and C). Indirect calorimetry assessed at 3 weeks after treatment revealed that ranolazine augments energy expenditure, as whole-body $\mathrm{O}_{2}$ consumption rates normalized to lean body mass were markedly increased (Figure 4A). Likewise, $\mathrm{O}_{2}$ consumption and $\mathrm{CO}_{2}$ production rates over 24 hours were higher in mice treated with ranolazine (Figure 4, B and C). Ranolazine treatment did not alter substrate preference, as respiratory exchange ratios were similar in lean or obese mice treated with ranolazine (Figure 4, D and E), whereas animal ambulatory activity was significantly reduced (Figure 4F).

Ranolazine treatment reverses obesity-induced hepatic steatosis. Liver mass normalized to body weight and hepatic triacylglycerol (TAG) content were both reduced in ranolazine-treated obese mice (Figure 5, A and $\mathrm{B}$ ), which were independent of reductions in circulating TAG and nonesterified fatty acid (NEFA) levels (Figure 5, C and D). In addition, frozen liver extracts were subjected to targeted metabolomic profiling for quantification of a broad range of acylcarnitines, which revealed no major changes except for increases in free carnitine (C0), C2, C4, and C18 acylcarnitines in obese mice treated with ranolazine (Supplemental Figure 1; supplemental material available online with this article; https://doi.org/10.1172/jci. insight.124643DS1). Ranolazine treatment also improved glycemia in response to a pyruvate tolerance test (Figure 5, E and F). Although ranolazine-mediated reductions in body weight likely contribute to our metabolic phenotypes, acute treatment of obese mice with ranolazine had no effect on pyruvate tolerance, hepatic TAG content, or body weight (Figure 6, A-C). Conversely, treatment of obese mice with ranolazine for 1 week improved glycemia during a pyruvate tolerance test and decreased hepatic TAG content, prior to any notable reductions in body weight (Figure 6, D-F).

Ranolazine increases hepatic PDH activity. Previous studies have demonstrated that ranolazine increases glucose oxidation (14). Therefore, examination of the phosphorylation of $\mathrm{PDH}$, the rate-limiting enzyme of glucose oxidation, revealed a marked reduction (indicative of increased $\mathrm{PDH}$ activity) in the livers of ranolazine-treated obese mice, which was associated with a reduction in hepatic $P d k 4$ but not $P d k 1 / 2 \mathrm{mRNA}$ expression (Figure 7, A and B). However, changes in hepatic $\mathrm{PDH}$ phosphorylation or Pdk1/2/4 expression were not observed in ranolazine-treated lean mice (Supplemental Figure 2). These ranolazine-mediated actions appear to be liver specific, as soleus muscles from obese mice and differentiated $\mathrm{C} 2 \mathrm{C} 12$ myotubes treated with ranolazine both demonstrated no changes in PDH phosphorylation (Supplemental Figure 3). Furthermore, PDH enzymatic activity was markedly increased in livers from obese mice treated with ranolazine (Figure 7C). Illustrating that these actions might be direct, we exposed HepG2 cells to $0.6 \mathrm{mM}$ oleate and $5.0 \mathrm{mM}$ glucose for 24 hours, followed by 24-hour treatment with $10 \mu \mathrm{M}$ ranolazine, which reduced both $\mathrm{PDH}$ phosphorylation and $P D K 4$ mRNA expression (Figure 7, D and E). As Pdk4 is a PPAR 


\section{O Lean VCO Lean Ran O Obese VC O Obese Ran}

A

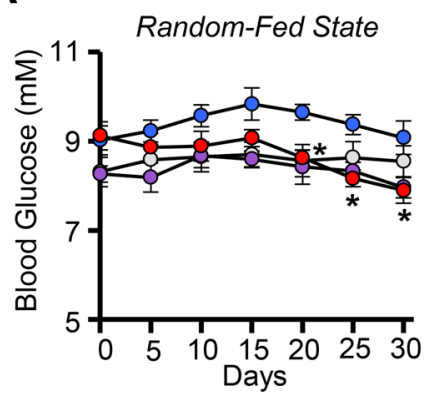

B

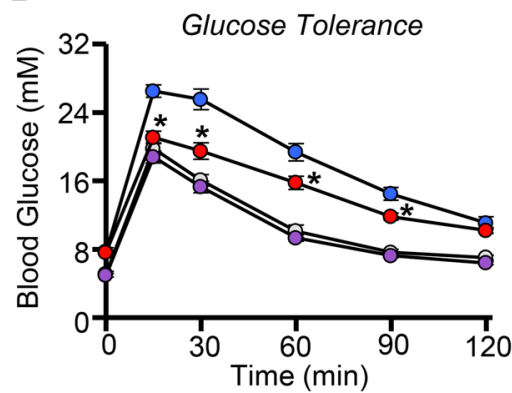

C

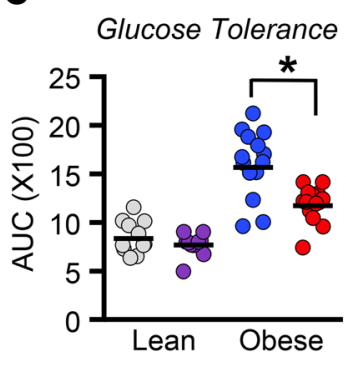

D

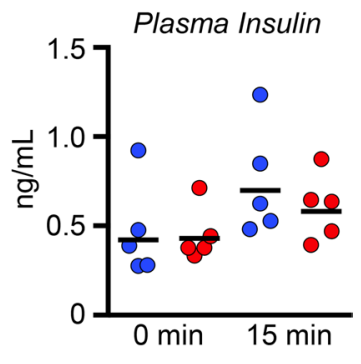

Figure 1. Ranolazine treatment improves glucose homeostasis. (A) Circulating glucose levels measured in lean and obese mice during the random-fed state over 30 days of vehicle control (VC) or ranolazine (Ran) treatment $(n=11-16)$. (B and C) Glucose tolerance in lean and obese mice treated with either VC or Ran; and AUC during the glucose tolerance test $(n=12-15)$. (D) Plasma insulin levels during the glucose tolerance test at 0 and 15 minutes after glucose administration $(n=5$ ). Values represent means \pm SEM. Differences were determined using a 2-way ANOVA, followed by a Bonferroni post hoc analysis. ${ }^{*} P<0.05$, significantly different from VC-treated counterpart.

downstream target in the liver (15), we evaluated the mRNA expression of additional PPAR $\alpha$ target genes (Slc27a1, Slc27a6, Acox1, Cd36, Acadm), a number of which were reduced in livers of obese but not lean mice treated with ranolazine (Supplemental Figure 4).

\section{Discussion}

Our study demonstrates that ranolazine mitigates hepatic steatosis, which may stem from ranolazine's ability to increase glucose oxidation, as ranolazine increased $\mathrm{PDH}$ activity in livers of obese mice and in HepG2 cells. Importantly, the ranolazine-mediated reduction in hepatic steatosis likely contributes to improved glycemic control, as ranolazine improved glucose and pyruvate tolerance in obese mice. Conversely, ranolazine failed to influence glycemia following either acute treatment in obese mice, or chronic treatment in lean mice, when minimal effects were observed on hepatic TAG content.

Our observations are of clinical relevance, as a significant fraction of patients with angina are also obese and comorbid for insulin resistance/T2D $(16,17)$. These individuals are, thus, likely to be receiving a combination of therapies to improve their angina symptoms, cardiac function, and dysglycemia. The US Food and Drug Administration (FDA) and European Medicines Agency have now mandated that new therapies in development for T2D must also be cardiovascular safe, as we do not want to simply improve glycemia in people with T2D but risk worsening their cardiovascular health (4). We would argue that it is equally prudent that we also ensure that our cardiovascular therapies do not worsen glucose homeostasis and T2D risk factors in angina patients comorbid for obesity/T2D. Indeed, studies have demonstrated that $\beta$-blockers, the first-line therapy for angina, may worsen glycemic control $(9,18)$. In addition, treatment with the $\beta$-blocker propranolol exacerbated nonalcoholic steatohepatitis (NASH) in C57BL/6J mice fed a methionine choline-deficient diet (19). On the contrary, ranolazine, which is a second-line agent for treating angina, has been shown in both preclinical and clinical studies to improve glycemia in animals and patients with T2D $(9,10)$. When combined with our observations demonstrating that ranolazine can reverse the progression of NAFLD, ranolazine would appear to be the more preferable antianginal therapy to consider in this specific patient population. If our observations in obese mice are recapitulated in obese humans, the translational implications of our findings could be quickly realized and easily implemented into clinical practice.

Studies from Dhalla and colleagues attempting to elucidate ranolazine's glycemia-lowering properties, demonstrated that ranolazine improves glycemia in rats via reducing glucagon secretion secondary to inhibiting the late $\mathrm{I}_{\mathrm{Na}}$ in islet $\alpha$-cells (10). Our findings in mice are not compatible with these assertions, as we observed no change in circulating glucagon levels during a pyruvate tolerance test (Supplemental Figure 5). Moreover, reduced glucagon secretion would not be compatible with ranolazine decreasing hepatic steatosis, since glucagon augments hepatic fatty acid oxidation rates and whole-body energy expenditure (20). Because of these positive actions of glucagon on energy expenditure, a number of studies have explored whether glucagon receptor agonism, or combining activation of both glucagon and glucagon-like peptide-1 receptors with new peptide coagonists, can be used as a novel approach to mitigate obesity (21). 


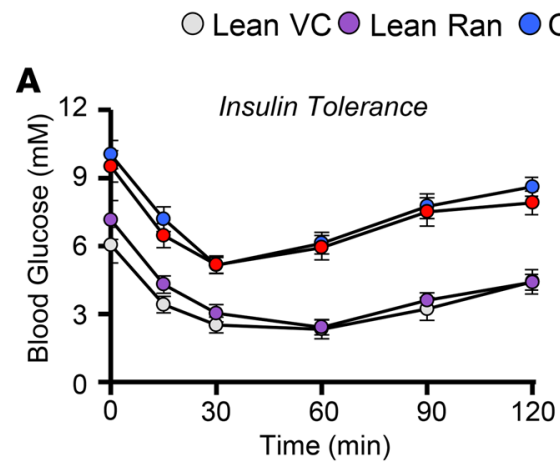

Obese VC O Obese Ran
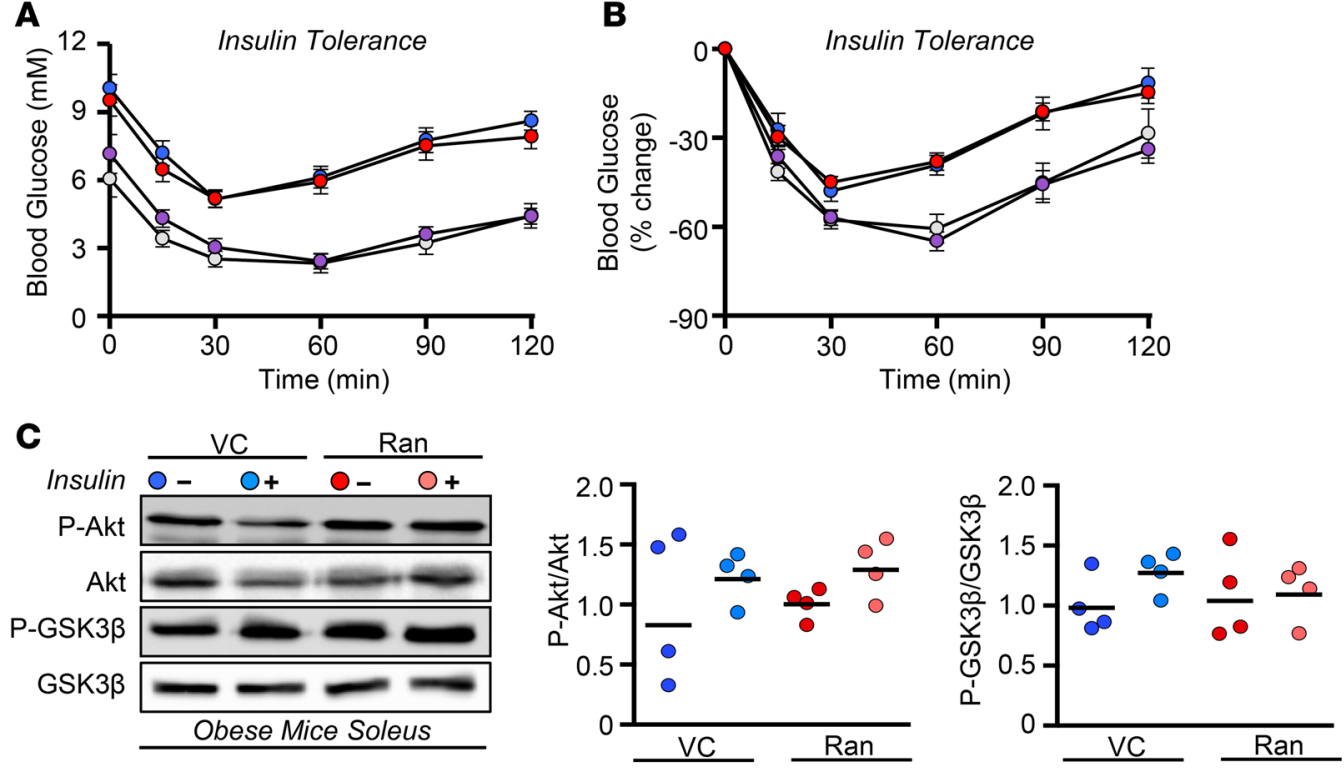

Figure 2. Ranolazine treatment does not influence insulin sensitivity. (A and B) Insulin tolerance in lean and obese mice treated with either vehicle control (VC) or ranolazine (Ran) ( $n=12-15)$. (C) Insulin signaling (Akt and GSK3 $\beta$ phosphorylation) in soleus from obese mice treated with either VC or $\operatorname{Ran}(n=4)$. Values represent means \pm SEM.

Reduced glucagon secretion and signaling would, in theory, worsen obesity-induced hepatic steatosis, and in support of that notion, whole-body glucagon receptor-deficient mice exhibit an exacerbation of obesity-induced NAFLD (20). Therefore, it is unlikely that the attenuation of NAFLD in response to treatment with ranolazine is dependent on reductions in circulating glucagon levels. It is also unlikely that ranolazine's cardiac mechanism of action, inhibition of the late $\mathrm{I}_{\mathrm{Na}}$, represents the mechanistic target by which ranolazine mitigates obesity-induced NAFLD. Ranolazine decreases the late $\mathrm{I}_{\mathrm{Na}}$ via inhibiting the voltage-gated $\mathrm{Na}$ channel subunit $1.5\left(\mathrm{Na}_{\mathrm{v}} 1.5\right)$ in the myocardium to improve angina symptoms, whereas $\mathrm{Na}_{\mathrm{v}} 1.5$ does not appear to be expressed to any appreciable extent within hepatocytes $(22,23)$.

Ranolazine's beneficial actions for angina were initially attributed to modulating energetics, since ranolazine increased PDH activity and glucose oxidation in both isolated skeletal muscle and the heart $(14,24,25)$. Our results are in support of these previous findings from McCormack and colleagues, as we observed that ranolazine treatment increased hepatic $\mathrm{PDH}$ activity in obese mice, suggesting that ranolazine also increases hepatic glucose oxidation. We further expand on these past observations by demonstrating that these actions
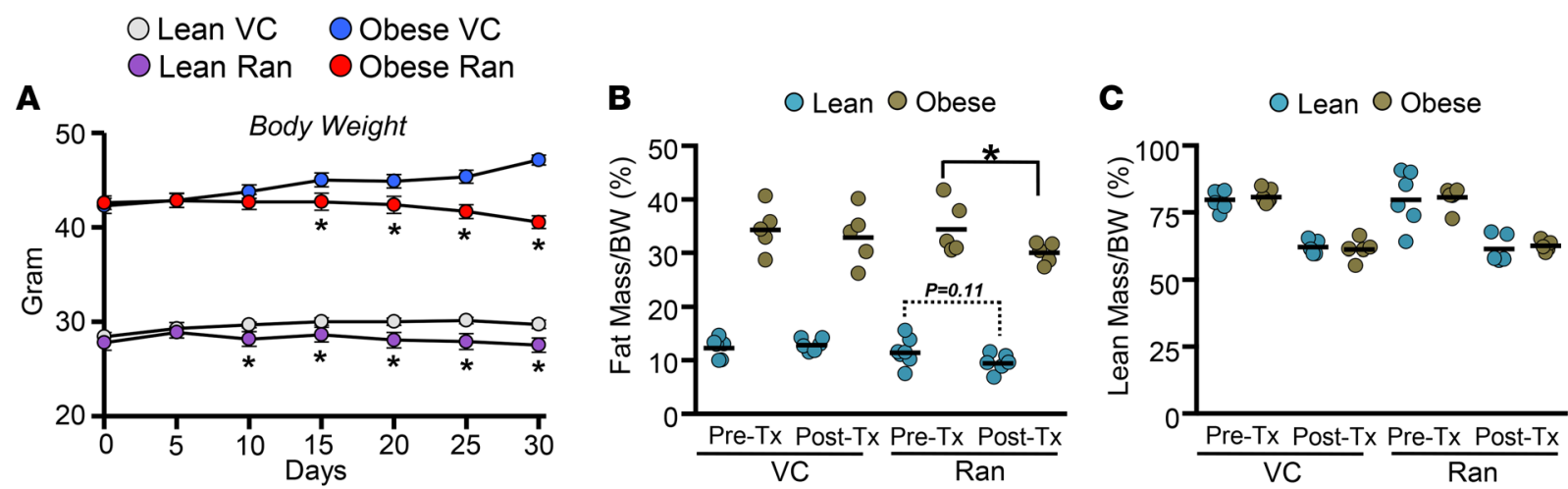

Figure 3. Ranolazine treatment reduces adiposity and decreases body weight gain. (A) Body weight measured over 30 days following vehicle control (VC) or ranolazine (Ran) treatment (Tx) in lean and obese mice $(n=11-16)$. (B and C) Body composition analysis in lean and obese mice treated with either VC or $\operatorname{Ran}(n=6-8)$. Values represent means \pm SEM. Differences were determined using a 2-way ANOVA, followed by a Bonferroni post hoc analysis. ${ }^{*} P<0.05$, significantly different from VC-treated counterpart. 


\section{$\bigcirc$ Lean VC $\bigcirc$ Lean Ran O Obese VC O Obese Ran}

A

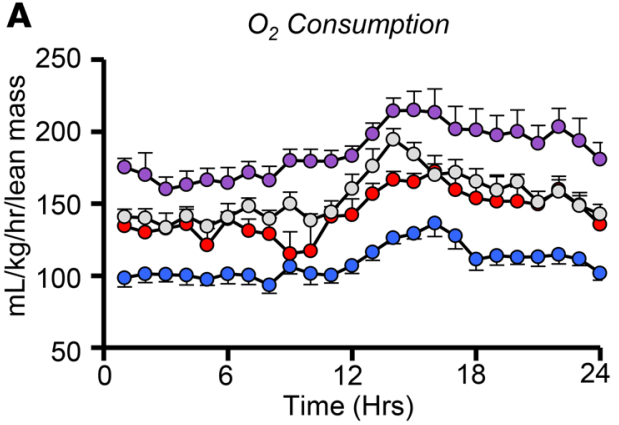

D

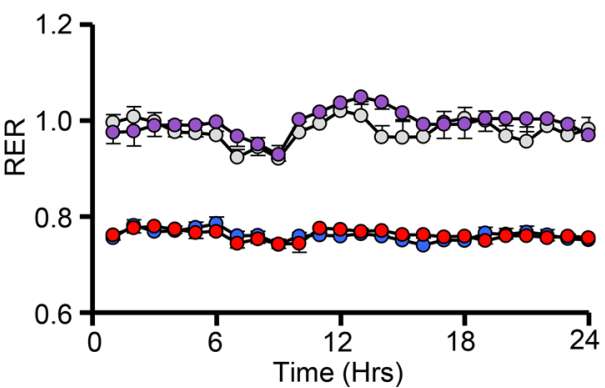

B

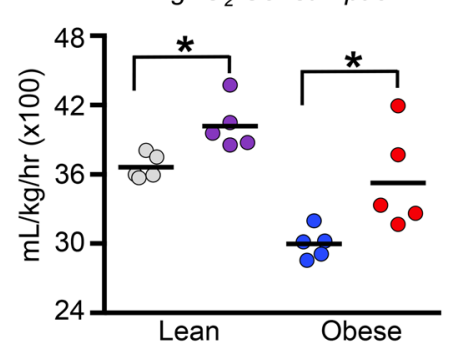

E

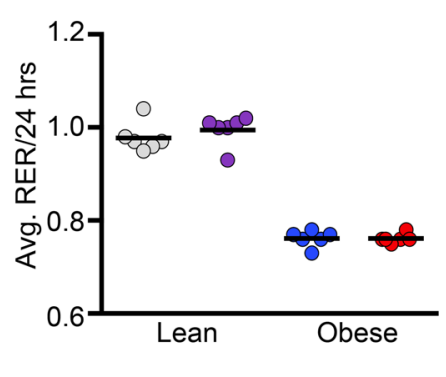

C Avg. $\mathrm{CO}_{2}$ Production

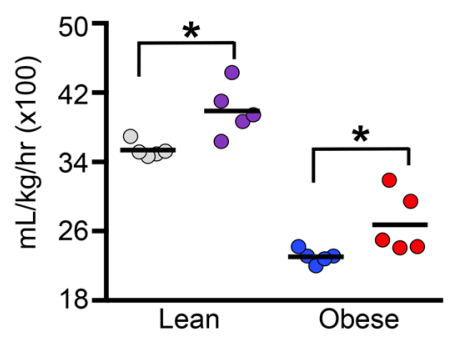

F Ambulatory Activity

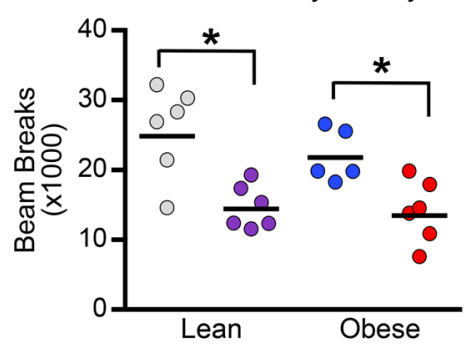

Figure 4. Ranolazine treatment improves whole-body oxygen consumption rates in obese mice. (A and B) Twenty-four-hour (light cycle from 0-12 hours; dark cycle from 12-24 hours) and average whole-body oxygen consumption rates in lean and obese mice treated with either vehicle control (VC) or ranolazine (Ran) $(n=5)$. (C) Average whole-body carbon dioxide production rates in lean and obese mice treated with either VC or Ran ( $n=5)$. ( $\mathbf{D}$ and $\mathbf{E})$ Twenty-four-hour (light cycle from 0-12 hours; dark cycle from 12-24 hours) and average respiratory exchange ratios (RER) in lean and obese mice treated with either VC or Ran $(n=5-6)$. (F) Twenty-four-hour ambulatory activity in lean and obese mice treated with either VC or Ran $(n=5-6)$. Values represent means \pm SEM. Differences were determined using a 2-way ANOVA, followed by a Bonferroni post hoc analysis. ${ }^{*} P<0.05$, significantly different from VC-treated counterpart.

of ranolazine contribute to ranolazine-mediated reductions in hepatic steatosis in obese mice. Of interest, a previous study demonstrated that elevated hepatic $\mathrm{PDH}$ activity via whole-body deficiency of Pdk2 confers substantial protection against obesity-induced NAFLD (12). In addition, reductions in hepatic steatosis were observed during either pharmacological treatment of obese mice with the liver-specific pan-PDHK inhibitor, 2-[(2,4-dihydroxyphenyl)sulfonyl]isoindoline-4,6-diol or in obese mice with a whole-body deficiency for $P d k 2$ and Pdk4 (13). This alleviation of hepatic steatosis was associated with reduced enzymatic activity of carbohydrate-responsive element binding protein and its downstream lipogenic target genes (Acaca, Fasn, and Scd1). Accordingly, it does appear that ranolazine-mediated increases in hepatic $\mathrm{PDH}$ activity may contribute to our phenotypic observations. However, the specific molecular mechanisms by which ranolazine increases hepatic PDH activity and subsequently attenuates NAFLD remain to be elucidated. Ranolazine also decreases fatty acid oxidation rates $(14,24)$, which could contribute to an increase in $\mathrm{PDH}$ activity/glucose oxidation via a Randle cycle mechanism $(26,27)$. On the contrary, impaired hepatic fatty acid oxidation has been proposed to be a major contributor to the pathology of hepatic steatosis/NAFLD $(28,29)$, making it unlikely that ranolazine increases hepatic PDH activity/glucose oxidation secondary to the inhibition of fatty acid oxidation.

An important consideration with our study is that NAFLD itself is relatively benign and not associated with significant pathology unless it progresses toward NASH. It is well characterized that experimental obesity in mice as a result of high-fat feeding does not produce any significant hepatic fibrosis and ballooning of hepatocytes associated with NASH over the time frame utilized in our study ( 30 , 31). Nevertheless, assessment of markers of inflammation in livers from obese mice treated with ranolazine demonstrated no major changes, other than a reduction in C-C motif chemokine ligand $2(C c l 2)$ (Supplemental Figure 6A). In addition, markers of fibrosis, including collagen type $1 \alpha 2$ chain (Colla2) and Colla1, trended to or were significantly reduced in livers from obese mice following treatment with ranolazine, suggesting that ranolazine may have salutary actions on hepatic fibrosis (Supplemental Figure 6B). Measurement of other markers of liver function, including circulating aspartate aminotransferase and alanine aminotransferase levels, remained unchanged in obese mice following treatment 

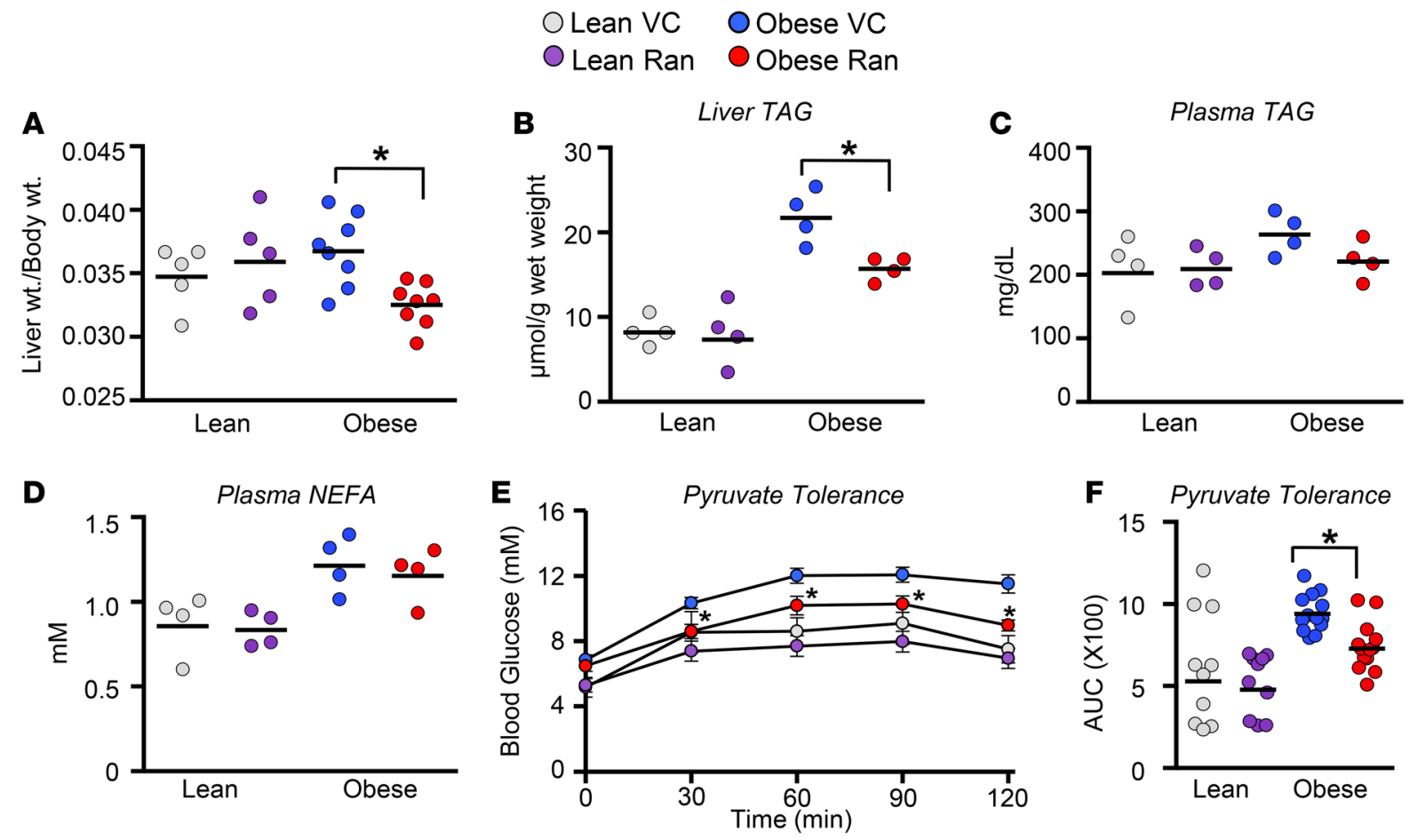

Figure 5. Ranolazine treatment reverses obesity-induced hepatic steatosis. (A) Liver weight/body weight ratios in lean and obese mice treated with either vehicle control (VC) or ranolazine (Ran) $(n=5-8)$. (B) Liver TAG content in lean and obese mice treated with either VC or Ran ( $n=4)$. (C and $\mathbf{D})$ Plasma TAG and NEFA levels in lean and obese mice treated with either VC or Ran $(n=4)$. (E and $\mathbf{F})$ Pyruvate tolerance in lean and obese mice treated with either VC or Ran, and the associated AUC ( $n=10-13)$. Values represent means \pm SEM. Differences were determined with a 2-way ANOVA, followed by a Bonferroni post hoc analysis. ${ }^{*} P<0.05$, significantly different from VC-treated counterpart.

with ranolazine (Supplemental Figure 7). Since one-third of patients with NAFLD will develop NASH with significant fibrosis, while NAFLD also remains a major risk factor for NASH in individuals with obesity and/or T2D $(32,33)$, our observations may thus suggest that ranolazine treatment could prevent or delay the progression to NASH in obese patients with angina. However, one must be cautious in interpreting these results due to the lack of fibrosis seen with high-fat feeding in mice, and it will be important for future studies to determine whether ranolazine can attenuate NASH pathology using the appropriate models.

Treatment with ranolazine also decreased adiposity and subsequent body weight gain in mice, which likely contributed to the improved glycemia and hepatic steatosis we observed in obese mice. Nonetheless, the increases in hepatic PDH activity, reversal of steatosis, and improved glycemia occurred prior to significant weight loss and in the absence of changes in circulating TAGs and NEFAs. Although further elucidation of the mechanisms by which ranolazine decreases adiposity/body weight in mice is of interest, clinical data in humans do not support these observations $(8,9)$, suggesting that our findings in mice may be species specific. Furthermore, the ranolazine-mediated reduction in body weight was independent of changes in both food and water intake (Supplemental Figure 8).

Taken together, ranolazine improves glycemia in experimental obesity, which may be due to both reductions in adiposity and hepatic steatosis. These improvements do not appear to be due to the classic target of ranolazine action, inhibition of the late $\mathrm{I}_{\mathrm{Na}}$, or reducing glucagon secretion, but they may involve enhanced hepatic PDH activity/glucose oxidation. Therefore, ranolazine may be a more preferable antianginal therapy to prescribe to obese individuals comorbid for angina, NAFLD, and T2D.

\section{Methods}

Animal care. Eleven-week-old male C57BL/6J mice were ordered from the Jackson Laboratory and allowed 1 week to acclimatize to the University of Alberta Health Sciences Laboratory Animal Services animal facility prior to study. Mice were placed on a standard chow/low-fat diet (10\% kcal from lard, Research Diets; D12450J) or high-fat diet (60\% kcal from lard, Research Diets; D12492) for a 10-week period. 
O Obese VC O Obese Ran
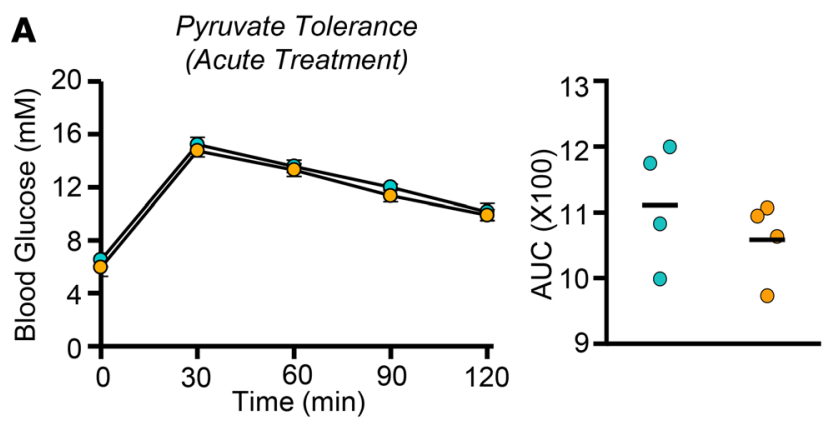

B
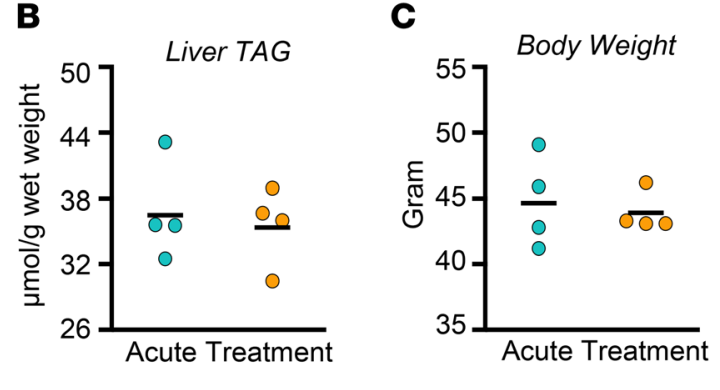

O Obese VC
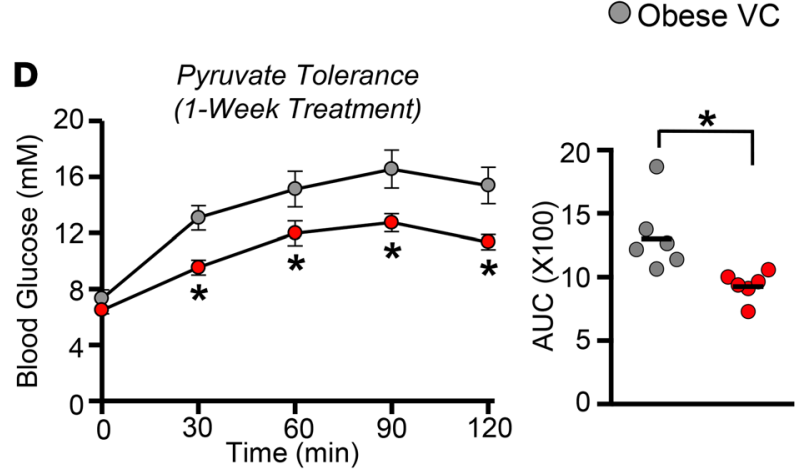

O Obese Ran

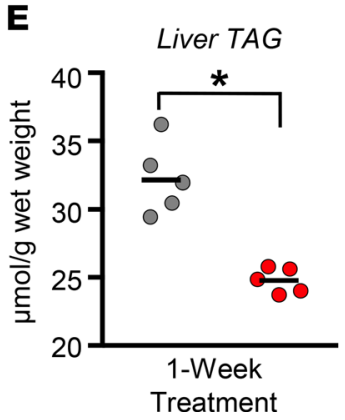

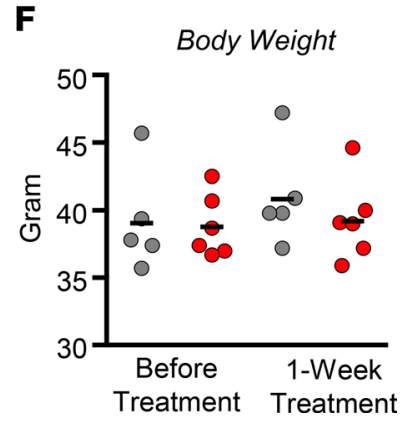

Figure 6. Ranolazine-mediated reductions in body weight are not required for ranolazine's salutary actions on NAFLD and dysglycemia. (A) Pyruvate tolerance and associated AUC. (B) Liver TAG content and (C) body weights in obese mice treated acutely with either vehicle control (VC) or ranolazine (Ran) $(n=4)$. (D) Pyruvate tolerance and associated AUC. (E) Liver TAG content and (F) body weights in obese mice treated with either VC or Ran for 1 week $(n=$ 5-6). Values represent means \pm SEM. Differences were determined with an unpaired, 2-tailed Student's $t$ test or a 2-way ANOVA, followed by a Bonferroni post hoc analysis. ${ }^{*} P<0.05$, significantly different from VC-treated counterpart.

At the end of week 10, animals underwent an i.p. glucose tolerance test, following which, all animals were randomized to receive treatment every day with ranolazine $(50 \mathrm{mg} / \mathrm{kg}$ ) or vehicle control (saline) via s.c. injection for 30 days. Animals were randomized in a manner that ensured that baseline glucose tolerance was not different between the saline- and ranolazine-treated groups. At the end of the study, animals were euthanized via i.p. injection of sodium pentobarbital $(12 \mathrm{mg})$ following an overnight fast and at 30 minutes after administration of saline or insulin for tissue extraction in liquid $\mathrm{N}_{2}$ with Wollenberger tongs. Separate cohorts of obese mice were treated $1 \times$ or for 1 week with saline or ranolazine $(50 \mathrm{mg} / \mathrm{kg})$ and euthanized upon completion of a pyruvate tolerance test following an overnight fast, after which, their livers were immediately extracted and snap frozen in liquid $\mathrm{N}_{2}$ using Wollenberger tongs.

Assessment of glucose homeostasis. Glucose, pyruvate, and insulin tolerance tests were performed in C57BL/6J mice as previously described (34). In brief, animals were fasted overnight; glucose, pyruvate, and insulin doses were $2 \mathrm{~g} / \mathrm{kg}, 2 \mathrm{~g} / \mathrm{kg}$, and $0.7 \mathrm{U} / \mathrm{kg}$, i.p., respectively. Blood glucose measurements were performed via tail bleed with the Accu-Chek Advantage system (Roche Diagnostics) at 0, 15, 30, 60, 90, and 120 minutes after glucose/pyruvate/insulin administration. Plasma was collected from tail whole-blood at 0 and 30 minutes of the glucose tolerance test, and circulating insulin levels were determined via use of a commercially available mouse ultrasensitive ELISA kit (Alpco Diagnostics, 80-INSMSU-E01) as previously described (35).

Indirect calorimetry. In vivo metabolic assessment via indirect calorimetry was performed using Oxymax metabolic cages (Columbus Instruments). Animals were initially acclimatized in the system for a 24-hour period, following which, the subsequent 24-hour period was utilized for data collection as previously described (34). The operator was blinded from mouse treatment group during the study and subsequent data acquisition.

Cell culture. All reagents were obtained from MilliporeSigma. C2C12 or HepG2 (American Type Culture Collection) cells were cultured in DMEM containing 10\% FBS and 1\% penicillin/streptomycin (P/S). Cells were incubated in a water-jacketed $\mathrm{CO}_{2}$ incubator maintained at $37^{\circ} \mathrm{C}$ with $5 \% \mathrm{CO}_{2}$. Upon confluency, 


\section{Obese VC O Obese Ran}
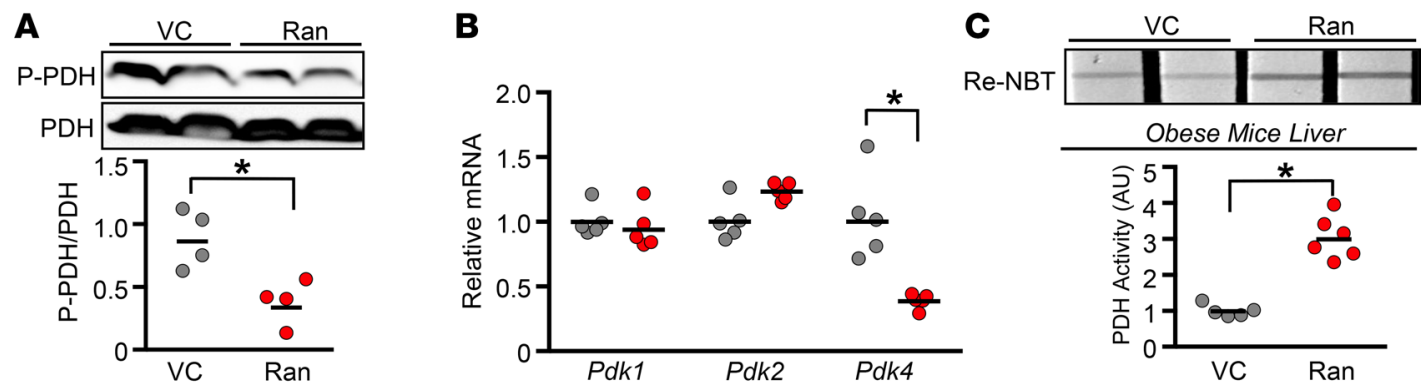

O HepG2 VC O HepG2 Ran
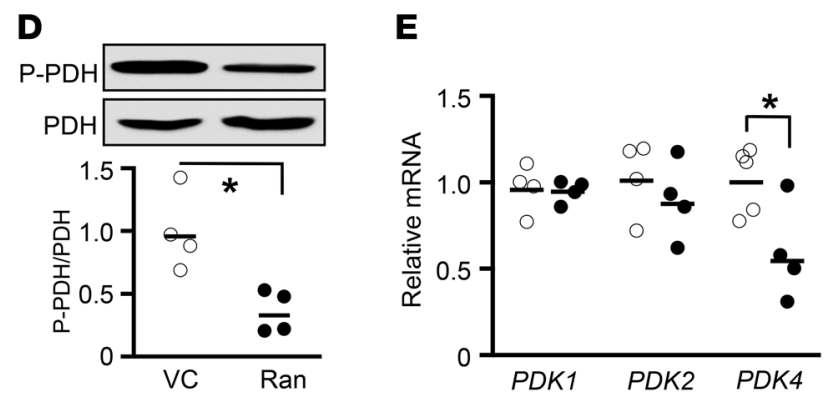

Figure 7. Ranolazine decreases hepatic PDH phosphorylation and Pdk4/PDK4 mRNA expression. (A) PDH phosphorylation (serine 293) in livers from obese mice treated with either vehicle control (VC) or ranolazine (Ran) for 1 week $(n=4)$. (B) Pdk mRNA expression in liver RNA extracts from obese mice treated with either VC or Ran for 1 week $(n=4-5)$. (C) PDH enzymatic activity in liver protein extracts from obese mice treated with either VC or Ran for 1 week $(n=5-6)$. (D) PDH phosphorylation (serine 293) in HepG2 cell treated with either VC or Ran $(10 \mu M)(n=4)$. (E) Pdk mRNA expression in HepG2 cell RNA extracts treated with either VC or Ran $(10 \mu \mathrm{M})(n=4-5)$. Values represent means \pm SEM. Differences were determined with an unpaired, 2-tailed Student's $t$ test. ${ }^{*} P<0.05$, significantly different from VC-treated counterpart.

C2C12 cells were differentiated into myotubes via growth in DMEM containing $2 \%$ horse serum and $1 \% \mathrm{P} / \mathrm{S}$ as previously described (36), following which, they were cultured in glucose free DMEM supplemented with $0.4 \mathrm{mM}$ oleate bound to $2 \%$ bovine serum albumin (BSA), $5.0 \mathrm{mM}$ glucose, $0.6 \mathrm{mM}$-hydroxybutyrate, 0.25 $\mathrm{mM}$ L-carnitine, and $0.2 \mathrm{nM}$ insulin. HepG2 cells were cultured in glucose free DMEM supplemented with $0.6 \mathrm{mM}$ oleate bound to $2 \% \mathrm{BSA}$ and $5.0 \mathrm{mM}$ glucose.

Western blotting. Frozen soleus or liver tissue (20 mg), or C2C12 myotubes or HepG2 cells, were homogenized in buffer containing $50 \mathrm{mM}$ Tris $\mathrm{HCl}\left(\mathrm{pH} 8\right.$ at $4^{\circ} \mathrm{C}$ ), $1 \mathrm{mM}$ EDTA, 10\% glycerol (w/v), $0.02 \%$ Brij-35 (w/v), 1 mM DTT, protease, and phosphatase inhibitors (MilliporeSigma), and protein samples were prepared and subjected to Western blotting protocols as previously described (37). Protein kinase B (Akt) and phospho-Akt (9272S and 4060L; Cell Signaling Technology); glycogen synthase kinase 3 (GSK3ß) and phospho-GSK3ß (5676S and 9331L; Cell Signaling Technology); PDH and phospho-PDH (3205S [Cell Signaling Technology] and ABS204 [MilliporeSigma]) antibodies were prepared in a 1/500 dilution in 5\% BSA.

Real-time PCR analysis. First-strand cDNA was synthesized from total RNA using the SuperScript III synthesis system (Invitrogen). Real-time PCR was carried out with the CFX connect Real time PCR machine (Bio-Rad) using TaqMan Gene Expression Assays (Applied Biosystems) or SYBR Green (Kapa Biosystems Inc.) (Supplemental Table 1). Relative mRNA transcript levels were quantified with the $2^{-\Delta \Delta C t}$ method using peptidylprolyl Isomerase A (Ppia/PPIA) or $\beta$-actin $(A c t b)$ as a housekeeping internal control gene as previously described (37).

PDH activity assay. PDH activity was measured in protein samples $(100 \mu \mathrm{g})$ extracted from frozen liver ( $\sim 40 \mathrm{mg}$ ) in obese mice using the MitoProfile Dipstick Assay Kit (MitoSciences) as previously described (37).

Blood biochemistry. Plasma samples collected from obese mice treated with vehicle control or ranolazine were used to detect levels of alanine aminotransferase, aspartate aminotransferase, and albumin using an EasyRA clinical chemistry analyzer (Medica).

Determination of hepatic and circulating TAG levels. Frozen powdered liver tissue $(\sim 20 \mathrm{mg})$ was extracted in a 2:1 chloroform/methanol solution, following which, the supernatant phase was retained for the assessment of TAG content with an enzymatic assay kit (Wako Pure Chemical Industries) as previously 
described (34). This same kit was also used to assess circulating TAG levels in $4 \mu \mathrm{l}$ plasma samples as previously described (34).

Metabolomic profiling. Approximately $50 \mathrm{mg}$ of frozen powdered liver tissue was subjected to a targeted quantitative metabolomics approach using a combination of direct injection mass spectrometry (DI-MS) with a reverse-phase liquid chromatography-MS/MS (LC-MS/MS) assay as previously described (38). The method used combines the derivatization and extraction of analytes, and the selective mass-spectrometric detection using multiple reaction monitoring pairs. Isotope-labeled internal standards are used for metabolite quantification. All the samples were thawed on ice and were vortexed and centrifuged at 13,000 $\mathrm{g}$. Each sample (10 $\mu \mathrm{l}$ of each) was loaded and dried in a stream of $\mathrm{N}_{2}$, following which, $20 \mu \mathrm{l}$ of a $5 \%$ solution of phenyl-isothiocyanate was added for derivatization. After incubation, samples were dried again using an evaporator. Extraction of the metabolites was then achieved by adding $300 \mu 1$ methanol containing $5 \mathrm{mM}$ ammonium acetate. The extracts were obtained by centrifugation at 13,000 $\mathrm{g}$, followed by a dilution step with kit MS running solvent. MS analysis was performed on an API4000 Qtrap MS/MS instrument (Applied Biosystems/MDS Analytical Technologies) equipped with a solvent delivery system. The samples were delivered to the mass spectrometer by a LC method followed by a DI method. Data analysis was performed and concentrations were calculated using Analyst software. The operator was blinded from sample ID treatment group during data acquisition and subsequent analysis.

Statistics. Values are presented as means \pm SEM. Significant differences were determined by the use of an unpaired, 2-tailed Student's $t$ test or a 2-way ANOVA followed by a Bonferroni post hoc analysis. All data were analyzed via GraphPad Prism 6.0 Software.

Study approval. All animals received care according to the guidelines developed by the Canadian Council on Animal Care. All experimental procedures performed in mice were approved by the University of Alberta Health Sciences Animal Welfare Committee.

\section{Author contributions}

$\mathrm{RA}$ and $\mathrm{KG}$ conceived and designed the experiments, performed the experiments, analyzed data, and wrote the paper. HA, AE, MA, AAG, SAC, BS, EAC, and FE performed the experiments, analyzed data, and provided discussions. PEL and GS provided discussions and reviewed/edited the paper. JRU conceived and designed the experiments and wrote the paper. JRU is the guarantor of this work, had full access to all the data, and takes responsibility for the integrity of the data and accuracy of the data analysis.

\section{Acknowledgments}

The authors would like to thank the dedicated staff of The Metabolomics Innovation Centre, where the acylcarnitine profiling was carried out. This study was supported by an Operating Grant from the Canadian Liver Foundation to JRU and project grants from the Canadian Institutes of Health Research (CIHR) to PEL and GS. RA is a fellow of the CIHR and Diabetes Canada. JRU is a Diabetes Canada Scholar. PEL holds the Dr. Charles A. Allard Chair in Diabetes Research.

Address correspondence to: John R. Ussher, 2-020E, Katz Centre for Pharmacy and Health Research, Faculty of Pharmacy and Pharmaceutical Sciences, University of Alberta, Edmonton, Alberta, T6G 2E1, Canada. Phone: 1.780.800.8391; Email: jussher@ualberta.ca.

1. Marchesini G, et al. Association of nonalcoholic fatty liver disease with insulin resistance. Am J Med. 1999;107(5):450-455.

2. Perry RJ, Samuel VT, Petersen KF, Shulman GI. The role of hepatic lipids in hepatic insulin resistance and type 2 diabetes. Nature. 2014;510(7503):84-91

3. Haas JT, Francque S, Staels B. Pathophysiology and Mechanisms of Nonalcoholic Fatty Liver Disease. Annu Rev Physiol. 2016;78:181-205.

4. Ussher JR, Sutendra G, Jaswal JS. The impact of current and novel anti-diabetic therapies on cardiovascular risk. Future Cardiol. 2012;8(6):895-912.

5. Drucker DJ, Goldfine AB. Cardiovascular safety and diabetes drug development. Lancet. 2011;377(9770):977-979

6. Sossalla S, Maier LS. Role of ranolazine in angina, heart failure, arrhythmias, and diabetes. Pharmacol Ther. 2012;133(3):311-323.

7. Undrovinas AI, Belardinelli L, Undrovinas NA, Sabbah HN. Ranolazine improves abnormal repolarization and contraction in left ventricular myocytes of dogs with heart failure by inhibiting late sodium current. J Cardiovasc Electrophysiol. 2006;17 Suppl 1:S169-S177.

8. Morrow DA, et al. Evaluation of the glycometabolic effects of ranolazine in patients with and without diabetes mellitus in the MERLIN-TIMI 36 randomized controlled trial. Circulation. 2009;119(15):2032-2039. 
9. Eckel RH, et al. Effect of Ranolazine Monotherapy on Glycemic Control in Subjects With Type 2 Diabetes. Diabetes Care. 2015;38(7):1189-1196.

10. Dhalla AK, et al. Blockade of $\mathrm{Na}+$ channels in pancreatic $\alpha$-cells has antidiabetic effects. Diabetes. 2014;63(10):3545-3556

11. Ussher JR, et al. Stimulation of glucose oxidation protects against acute myocardial infarction and reperfusion injury. Cardiovasc Res. 2012;94(2):359-369.

12. Go Y, et al. Inhibition of Pyruvate Dehydrogenase Kinase 2 Protects Against Hepatic Steatosis Through Modulation of Tricarboxylic Acid Cycle Anaplerosis and Ketogenesis. Diabetes. 2016;65(10):2876-2887.

13. Wu CY, et al. Targeting hepatic pyruvate dehydrogenase kinases restores insulin signaling and mitigates ChREBP-mediated lipogenesis in diet-induced obese mice. Mol Metab. 2018;12:12-24.

14. McCormack JG, Barr RL, Wolff AA, Lopaschuk GD. Ranolazine stimulates glucose oxidation in normoxic, ischemic, and reperfused ischemic rat hearts. Circulation. 1996;93(1):135-142.

15. Kersten S. Integrated physiology and systems biology of PPAR $\alpha$. Mol Metab. 2014;3(4):354-371.

16. Borgeraas $\mathrm{H}$, et al. Association of body mass index with risk of acute myocardial infarction and mortality in Norwegian male and female patients with suspected stable angina pectoris: a prospective cohort study. BMC Cardiovasc Disord. 2014;14:68.

17. Shukla A, Kumar K, Singh A. Association between obesity and selected morbidities: a study of BRICS countries. PLoS ONE. 2014;9(4):e94433.

18. Rizos CV, Elisaf MS. Antihypertensive drugs and glucose metabolism. World J Cardiol. 2014;6(7):517-530.

19. McKee C, et al. Propranolol, a $\beta$-adrenoceptor antagonist, worsens liver injury in a model of non-alcoholic steatohepatitis. Biochem Biophys Res Commun. 2013;437(4):597-602.

20. Longuet C, et al. The glucagon receptor is required for the adaptive metabolic response to fasting. Cell Metab. 2008;8(5):359-371.

21. Capozzi ME, DiMarchi RD, Tschöp MH, Finan B, Campbell JE. Targeting the Incretin/Glucagon System With Triagonists to Treat Diabetes. Endocr Rev. 2018;39(5):719-738.

22. Nuyens D. Generation and Characterization of a Mouse Model for a Sodium Channel Based Long QT Syndrome (LQT3), an Inherited Arrhythmogenic Disease. Leuven, Belgium: Leuven University Press; 2006.

23. SCN5A. The Human Protein Atlas Web site. https://www.proteinatlas.org/ENSG00000183873-SCN5A/tissue. Accessed December 7, 2018.

24. McCormack JG, Baracos VE, Barr R, Lopaschuk GD. Effects of ranolazine on oxidative substrate preference in epitrochlearis muscle. J Appl Physiol. 1996;81(2):905-910.

25. Clarke B, Spedding M, Patmore L, McCormack JG. Protective effects of ranolazine in guinea-pig hearts during low-flow ischaemia and their association with increases in active pyruvate dehydrogenase. Br J Pharmacol. 1993;109(3):748-750.

26. Hue L, Taegtmeyer H. The Randle cycle revisited: a new head for an old hat. Am J Physiol Endocrinol Metab. 2009;297(3):E578-E591.

27. Lopaschuk GD, Ussher JR, Folmes CD, Jaswal JS, Stanley WC. Myocardial fatty acid metabolism in health and disease. Physiol Rev. 2010;90(1):207-258.

28. Savage DB, Petersen KF, Shulman GI. Disordered lipid metabolism and the pathogenesis of insulin resistance. Physiol Rev. 2007;87(2):507-520.

29. Zhang D, et al. Mitochondrial dysfunction due to long-chain Acyl-CoA dehydrogenase deficiency causes hepatic steatosis and hepatic insulin resistance. Proc Natl Acad Sci USA. 2007;104(43):17075-17080.

30. Kohli R, Feldstein AE. NASH animal models: are we there yet? J Hepatol. 2011;55(4):941-943.

31. Lau JK, Zhang X, Yu J. Animal models of non-alcoholic fatty liver disease: current perspectives and recent advances. J Pathol. 2017;241(1):36-44.

32. Farrell GC, Larter CZ. Nonalcoholic fatty liver disease: from steatosis to cirrhosis. Hepatology. 2006;43(2 Suppl 1):S99-S112.

33. McPherson S, Hardy T, Henderson E, Burt AD, Day CP, Anstee QM. Evidence of NAFLD progression from steatosis to fibrosing-steatohepatitis using paired biopsies: implications for prognosis and clinical management. J Hepatol. 2015;62(5):1148-1155.

34. Ussher JR, et al. Inhibition of de novo ceramide synthesis reverses diet-induced insulin resistance and enhances whole-body oxygen consumption. Diabetes. 2010;59(10):2453-2464.

35. Ussher JR, et al. Genetic and Pharmacological Inhibition of Malonyl CoA Decarboxylase Does Not Exacerbate Age-Related Insulin Resistance in Mice. Diabetes. 2016;65(7):1883-1891.

36. Ussher JR, et al. Role of the atypical protein kinase Czeta in regulation of 5'-AMP-activated protein kinase in cardiac and skeletal muscle. Am J Physiol Endocrinol Metab. 2009;297(2):E349-E357.

37. Gopal K, et al. FoxO1 regulates myocardial glucose oxidation rates via transcriptional control of pyruvate dehydrogenase kinase 4 expression. Am J Physiol Heart Circ Physiol. 2017;313(3):H479-H490.

38. Sung MM, et al. Improved Glucose Homeostasis in Obese Mice Treated With Resveratrol Is Associated With Alterations in the Gut Microbiome. Diabetes. 2017;66(2):418-425. 